Esta sección esta abierta para todos los lectores de la Revista, a la que pueden enviar sus preguntas, comentarios o críticas a los artículos que hayan sido publicados en los últimos números, teniendo en cuenta la posibilidad de que los autores aludidos puedan responder. Podrá aceptarse la comunicación de investigaciones preliminares, o de intervenciones en brotes que no hayan sido publicados ni sometidos a publicación en otra revista; así como, algunos comentarios sobre problemas de salud pública, ética y educación médica. La extensión máxima aceptable es de 1000 palabras, con un máximo de seis referencias bibliográficas (incluyendo la referencia del artículo que la motivó, cuando sea el caso) y una tabla o figura. Esta puede ser enviada a revmedex@ins.gob.pe.

\section{INTENCIÓN EN LA MODALIDAD DE TITULACIÓN DE INTERNOS DE MEDICINA EN EL PERÚ, 2010}

\section{INTENTION IN THE WAY OF GETTING THE MEDICAL DIPLOMA AMONG MEDICAL INTERNS IN PERU, 2010}

\author{
Edén Galán-Rodas ${ }^{1, a}$, Cristian Díaz-Vélez ${ }^{1,2, b}$, \\ Christian R. Mejia1, 3,a
}

Sr. Editor. En el Volumen 27, Número 3 de su revista, resaltamos que la temática central haya estado dirigida a la importancia de la investigación en salud, considerando que dicha actividad en medicina no solo es una tarea universitaria obligatoria para la obtención de nuevos conocimientos sino que constituye el eje de la formación médica. Al respecto, en el año 2001 se publicó un estudio sobre el quehacer de los investigadores de la Facultad de Medicina de la Universidad Nacional Mayor de San Marcos, el cual reportó que el $41 \%$ de los investigadores médicos se inició como tal siendo aún estudiante de pregrado ${ }^{(1)}$.

Sin embargo, en 1991 una norma legal afectó -quizá sin proponérselo- la realización de tesis, pues mediante el Decreto Legislativo 739 se aprobó el bachillerato automático, se consideraba, además, que el título profesional se podría obtener de tres modos: a) Presentación y aprobación de tesis; b) Después de ser egresado y haber prestado servicios profesionales durante tres años consecutivos en labores propias de la especialidad, debiendo presentar un trabajo u otro documento a

Comité del Médico Joven. Colegio Médico del Perú. Lima, Perú.

2 Hospital Nacional Almanzor Aguinaga Asenjo ESSALUD. Chiclayo, Perú.

3 Escuela de Inteligencia del Ejército. Lima, Perú.

a Médico; b Médico Epidemiólogo.

Recibido: 14-02-11 Aprobado: 16-02-11 criterio de la Universidad; y c) Cualquier otra modalidad que estime conveniente la universidad. Esta norma que fue aplicada por muchas facultades de medicina, recién a partir del 2003 propició una disminución importante en la realización de tesis para optar el título profesional ${ }^{(2)}$.

Ante esta situación, el 10 de agosto de 2009, el directorio del Consejo de Evaluación, Acreditación y Certificación de la Calidad de la Educación Superior Universitaria (CONEAU), aprobó el modelo de calidad para la acreditación de la carrera profesional universitaria de Medicina que consta de tres dimensiones, nueve factores, 16 criterios y 98 estándares. En la dimensión de formación profesional, dentro del factor de enseñanza-aprendizaje, se observa que uno de los estándares exige que el $75 \%$ de titulaciones deben obtenerse mediante tesis; por otro lado en cuanto al factor de investigación se observa que cuenta con diez estándares que, en resumen, pretenden evaluar la participación de los estudiantes en investigaciones reconocidas por la facultad, eventos de discusión, difusión y publicación de los resultados ${ }^{(3)}$. Estos nuevos estándares de calidad permitirán que el interés por la investigación se incremente, a diferencia de los estándares mínimos de acreditación otorgados por la Comisión de Acreditación de Facultades de Medicina (CAFME), en los cuales se mencionaba que se debía contar con políticas y líneas de investigación aprobadas y la exigencia de solo el $2 \%$ de los créditos de la malla curricular destinados a cursos de metodología de la investigación y estadística ${ }^{(4)}$.

Entre junio y noviembre del año 2010, el Comité Médico Joven del Colegio Médico del Perú, aplicó un cuestionario a 634 internos de medicina procedentes de 26 escuelas y facultades de medicina del país, en 12 ciudades (Piura, Cajamarca, Huánuco, Chiclayo, Trujillo, Lima, Arequipa, Cusco, Ica, Chimbote, Ayacucho y Tacna), de donde se obtuvo una tasa de rechazo menor a 1\%. En el cuestionario se indagaba sobre las actividades que realizan los estudiantes durante el internado de medicina: académicas, laborales, sociales y de investigación, dentro de esta última la intención en la modalidad de titulación. 
Del total de internos de medicina de universidades peruanas, el $52,3 \%$ provenían de escuelas de medicina donde tienen más de una opción para obtener el título profesional como la tesis, el examen de suficiencia o de grado y el curso de titulación. Analizamos la intención de titulación en este grupo, en el cual el 56,9\% fue de sexo masculino y la media de edad 24,9 años (rango: $21-41$ años), encontramos que un $49,1 \%$ tiene intención de titularse por la modalidad de tesis, $21,5 \%$ por el curso de titulación y $29,4 \%$ por examen de suficiencia.

Estos resultados difieren del estudio realizado en el 2007 a 202 internos de medicina de la Universidad Nacional Mayor de San Marcos, el cual reveló solo un 4,6\% de intención de titulación por la modalidad de tesis ${ }^{(5)}$. En nuestro estudio, esa intención alcanza el $11 \%$ entre quienes realizan el internado de medicina. Esta marcada diferencia podríamos atribuirla al rol de instituciones como el Instituto Nacional de Salud en el fortalecimiento del Sistema Nacional de Investigación en Salud y a las estrategias implementadas para tal fin; entre ellas, resaltar la indexación en la Base de datos MEDLINE de la Revista Peruana de Medicina Experimental y Salud Pública, que significa un gran paso en la difusión de la producción científica peruana y, en especial, por la apertura en la publicación de artículos estudiantiles.

Otro rol importante lo cumple el Colegio Médico del Perú con el auspicio y apoyo a los eventos científicos estudiantiles que año tras año organiza la Sociedad Científica Médico Estudiantil Peruana, SOCIMEP, (antes SOCIPEM), organización fundada originalmente en 1992 y refundada por conceptos legales en el 2007. SOCIMEP, cuenta con 31 sociedades científicas estudiantiles afiliadas a nivel nacional, que en los últimos años ha emprendido y generado cambios en sus políticas de fomento a la investigación científica en pregrado ${ }^{(5)}$, lo que ha permitido motivar a un mayor número de estudiantes a participar de actividades de investigación con trabajos colaborativos, predominantemente en áreas de salud pública y educación médica que han permitido, a la fecha, numerosas publicaciones estudiantiles en revistas indexadas y un mayor compromiso con esta noble labor inherente de todo estudiante universitario. Esto, a la par con los nuevos cambios generados por el CONEAU y el incremento progresivo del número de egresados con la intención de obtener el título profesional bajo la modalidad de tesis, nos permite avizorar un futuro prometedor en la investigación científica de nuestro país.

\section{Conflictos de Interés}

EGR, CDV y CRM son ex-miembros de la Sociedad Científica Médica Estudiantil Peruana, SOCIMEP. (Antes SOCIPEM).

\section{REFERENCIAS BIBLIOGRÁFICAS}

1. Sogi C, Perales A. El Quehacer de los Investigadores de la Facultad de Medicina. An Fac med. 2001;62(2):100-14.

2. Góngora M. El Estado, la universidad peruana y el drama de la investigación científica. Escritura y Pensamiento. 2002;5(11):43-57.

3. Consejo de Evaluación, Acreditación y Certificación de la Calidad de la Educación Superior Universitaria (CONEAU). Modelo de calidad para la acreditación de la carrera profesional universitaria de Medicina. [Separata Especial]. Lima: Diario Oficial El Peruano; 25 de noviembre 2009.

4. Comisión para la Acreditación de Facultades o Escuelas de Medicina Humana (CAFME). Resolución Suprema №07-2006-SA. Actualización de Estándares Mínimos para la Acreditación de Facultades o Escuelas de Medicina. Lima: CAFME; 2006.

5. Huamaní $C$, Chávez-Solís $\mathbf{P}$, Domínguez-Haro W, Solano-Aldana M. Producción científica estudiantil: análisis y expectativas. Rev Peru Med Exp Salud Publica. 2007;24(4):444-6.
Correspondencia: Edén Galán Rodas

Dirección: Av. Malecón Armendáriz N. ${ }^{\circ} 791$, Lima 18, Perú.

Teléfono: (511) 986-653590

Correo electrónico: edgarod6@gmail.com

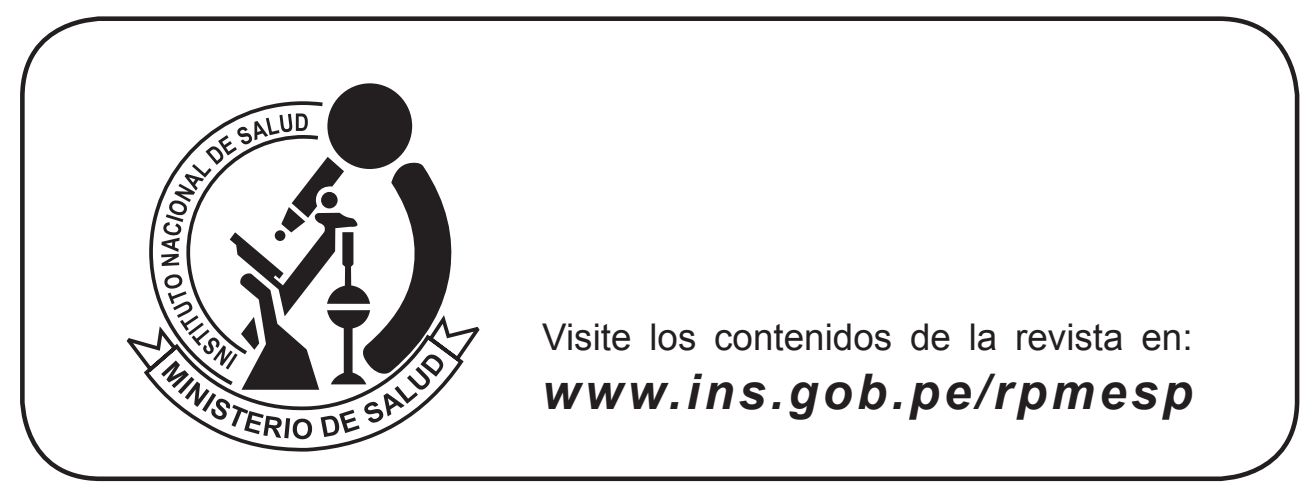

